\title{
Elaboración de bloques de construcción a partir de residuos de arena sílica proveniente del proceso de fundición aluminio en una empresa automotriz
}

\section{Preparation of construction blocks from silicone sand waste from the aluminum foundation process in an automotive company}

GÓMEZ-BELTRÁN, Guillermina†*, SIERRA-SALGADO, Diana Vanessa y MARTÍN DEL CAMPOSÁNCHEZ, Ma. Guadalupe

Universidad Tecnológica del Valle de Toluca

ID $1^{\text {er }}$ Autor: Guillermina, Gómez-Beltrán / ORC ID: 0000-0002-3621-2262, CVU CONACYT ID: 93419

ID $1^{\text {er }}$ Coautor: Diana Vanessa, Sierra-Salgado / ORC ID: 0000-0002-1737-2172

ID $2^{\text {do }}$ Coautor: Ma. Guadalupe, Martín Del Campo-Sánchez / ORC ID: 0000-0003-2689-1684

DOI: $10.35429 / J C E .2019 .10 .3 .19 .26$

Recibido 03 de Septiembre, 2019; Aceptado 30 Diciembre, 2019

\section{Resumen}

El objetivo del proyecto fue desarrollar un procedimiento para la elaboración de bloques de construcción con residuos de arena sílica, provenientes de un proceso de fundición del aluminio de una empresa de giro automotriz. Los bloques obtenidos se evaluaron de conformidad a las normas NMX-C-404-1997-ONNCCE para el diseño y formulaciones del block, y la NMX-C-036-ONNCCE para las pruebas y cálculos de compresión. Inicialmente estas arenas son utilizadas para la elaboración de los moldes de las partes mecánicas que se fabrican en la empresa, sin embargo, una vez que se cumple la utilidad de estas arenas para moldeo, son enviadas a sitios de disposición final. Para la elaboración de los bloques, inicialmente se determinaron las características físicas y propiedades químicas de la arena sílica (tamaño de grano, pH, estructura, forma del grano, humedad, peso específico, densidad aparente y a granel). A continuación, se diseñó el molde y se probaron dos formulaciones a base de los residuos de arena sílica, cemento, agua y cal. La evaluación de la calidad del producto incluyó las pruebas de secado, absorción de humedad, resistencia de diseño a la compresión. Finalmente se compararon los costos de elaboración con los costos de bloques comerciales existentes en el mercado.

Bloque, Residuo, Arena Sílica

\begin{abstract}
The objective of the project was to develop a procedure for the construction of building blocks with silica sand residues, from an aluminum smelting process of an automotive spinning company. The blocks obtained were evaluated in accordance with NMX-C-404-1997ONNCCE standards for the design and formulations of the block, and NMX-C-036-ONNCCE for tests and compression calculations. Initially these arenas are used for the elaboration of the molds of the mechanical parts that are manufactured in the company, however, once the utility of these arenas for molding is fulfilled, they are sent to final disposal sites. For the elaboration of the blocks, the physical characteristics and chemical properties of the silica sand were initially determined (grain size, $\mathrm{pH}$, structure, grain shape, humidity, specific gravity, bulk and bulk density). The mold was then designed and two formulations based on the residues of silica sand, cement, water and lime were tested. The product quality assessment included tests for drying, moisture absorption, design resistance to compression. Finally, the manufacturing costs were compared with the costs of existing commercial blocks in the market.
\end{abstract}

Block, Residue, Silica Sand

Citación: GÓMEZ-BELTRÁN, Guillermina, SIERRA-SALGADO, Diana Vanessa y MARTÍN DEL CAMPO-SÁNCHEZ, Ma. Guadalupe. Elaboración de bloques de construcción a partir de residuos de arena sílica proveniente del proceso de fundición aluminio en una empresa automotriz. Revista de Ingeniería Civil. 2019. 3-10: 19-26

\footnotetext{
* Correspondencia del Autor (correo electrónico: guillermina.gomez@utvtol.edu.mx)

$\dagger$ Investigador contribuyendo como primer autor.
} 


\section{Introducción}

Para la fabricación de productos de origen metálico, la fundición es una de las tecnologías principales utilizadas. Esta tecnología consiste en una serie de operaciones mediante las cuales se obtiene un molde que reproduce la forma de la pieza a fabricar, en el que se vierte el metal en el interior de un molde previamente fabricado. Se emplea fundamentalmente en piezas como motores, cigüeñales, componentes de turbinas hidráulicas entre otras (Herrera, 2011).

El material del molde marca en gran medida, las características generales del proceso de fundición. Dentro de la empresa donde se desarrolló el presente proyecto, se utilizan componentes de origen natural y sintético para la producción del molde de los productos elaborados en la misma, los cuales están formados principalmente de arena de sílice y una aplicación de amina como resina, lo que permite el aglutinamiento del material.

Estos moldes son descartados una vez que han cumplido con su función. Debido a ello, uno de los principales problemas a los que se ha enfrentado el sistema productivo dentro de la empresa, ha sido la gran generación de residuos de arena sílica. Una vez que se cumple la utilidad de estas arenas para moldeo, son recolectadas y depositadas en súper sacos con una capacidad de aproximadamente una tonelada, posteriormente son distribuidos hacia otras organizaciones quienes se encargan de destinar estos residuos en un sitio de disposición final adecuado según sean sus características químicas y físicas.

En esta situación, la reducción de la generación es deseable, pero es poco probable de implementar en la empresa, por las exigencias productivas de la misma. La Ley General para la Prevención y Gestión Integral de Residuos (LGPGIR) establece las acciones de valorización de los residuos como de orden público e interés social y que tienen por objeto garantizar el derecho de toda persona al medio ambiente sano y a propiciar el desarrollo sustentable (DOF, 2003). Los materiales industriales tales como las arenas de moldeo generados en la empresa son productos valorables de procesos industriales, que pueden ser ampliamente utilizados en la industria de la construcción.
En la actualidad, se considera e incluso se lleva a cabo la construcción de diferentes tipos de edificaciones con gran variedad de materiales, es probable que se encuentren algunos que sean más adecuados que otros, sin embargo, se hace necesario conocer su procedencia, pues tratándose de materiales reciclados, sería preciso pensar en ciertos factores como su durabilidad y la calidad de este mismo, ya que los materiales presentan diversas cualidades que determinan el tiempo de vigencia de cualquier construcción.

Los usos y aplicaciones de la arena sílica, se derivan principalmente por sus propiedades químicas y físicas, entre los que destacan su dureza, resistencia química, piezoelectricidad, alto punto de fusión, transparencia $\mathrm{y}$ piroelectricidad.

Debido a lo anterior, en el presente proyecto se propuso cuantificar y caracterizar los residuos de arena sílica se generan dentro de la empresa, asimismo, desarrollar una metodología para la fabricación de tabiques de construcción que cumplan con las especificaciones y parámetros de construcción utilizando como materia prima los residuos de arena sílica que se genera en el área de fundición aluminio de la empresa. Estos tabicones fueron elaborados a partir de diferentes formulaciones a base de arena sílica, cemento gris, cal y agua. Una vez obtenidos los tabicones, se evaluaron sus características físicas que permitieron identificar la mezcla con mejores características.

Adicionalmente se realizó un estudio de costo beneficio para establecer la factibilidad de sustituir el material comúnmente utilizado por los pequeños fabricantes de tabicón y verificar si es posible la reducción en los costos de producción.

1. Descripción de proceso de generación del residuo de arena sílica

La empresa cuenta con un área denominada "Zona de Colectores", donde se recolectan y recuperan las arenas después de que han sido sometidas a un tratamiento térmico para posteriormente ser enviadas a los diferentes silos de acumulación. El funcionamiento mecánico inicial, consiste en volcar la arena que se va a regenerar, a una altura de tres metros, hacía una tolva de carga ubicada en la parte superior de un transportador vibratorio. 
Del transportador, la arena pasa a una trituradora rotativa, la cual permite reducir la granulometría del producto donde es descartado el material extraño de la arena y posteriormente se descarga en un contenedor. Por medio de un sinfín, la arena es tomada e introducida en el horno de regeneración a una temperatura de 600$800^{\circ} \mathrm{C}$. La combustión en el interior del horno está asegurada por un quemador piloto con detector de llama por ionización que es mantenido constantemente encendido por motivos de seguridad.

Al finalizar la calcinación, el producto pasa por una cámara de enfriamiento, donde por medio de gravedad, la arena regenerada es separada, y a través de un equipo de aspiración se separan las partículas pequeñas (arena residual) hacia los llamados super sacos. Las arenas residuales se caracterizan por tener un tamaño de grano muy pequeño que no le permite su reutilización en la fabricación de moldes para la fundición y son el objeto de estudio del presente trabajo.

\section{Materiales y métodos}

Como primer paso, se cuantificaron las cantidades mensuales generadas del residuo de arena sílica. Posteriormente se procedió a realizar un muestreo de los residuos de arena sílica. Se obtuvieron seis muestras simples tomadas semanalmente durante 2 meses. De manera individual, se determinaron algunos parámetros físicos y mecánicos para caracterizar al material: granulometría, $\mathrm{pH}$, estructura, forma del grano, humedad, peso específico, densidad aparente y densidad a granel.

Para el diseño del molde de madera se respetaron las dimensiones especificadas en la norma NMX-C-404-1997-ONNCCE para la fabricación del tabique $(24 X 14 \times 10 \mathrm{~cm})$. A continuación, prepararon dos formulaciones, la primera consistió en una mezcla de arena sílica con cemento y agua, y la segunda en una mezcla de arena sílica, cemento, agua y cal. Ambas fueron vertidas en los moldes (por triplicado) y se analizaron sus propiedades resistencia al diseño de compresión y capacidad de absorción de agua, posteriores al secado del tabicón.

La resistencia de diseño a la compresión se obtuvo a partir de tablas de referencia establecidas en la norma antes mencionada. El ensayo de capacidad de absorción se efectuó inmediato al periodo de secado.
Para ello, los tabiques secos se pesaron de manera individual (peso en seco) y se introdujeron a un tanque con agua de modo que quedaran totalmente cubiertos por el líquido y que ésta pudiera circular libremente alrededor del tabique. Después de un periodo de 24 horas, se retiraron del agua y se pasó un trapo húmedo sobre su superficie para quitar el exceso de agua. De inmediato se pesaron (peso saturado) y se calculó la capacidad de absorción de agua. Los datos obtenidos se compararon con los reportados para diferentes materiales de construcción y se identificó la formulación con mejores características.

Finalmente, se compararon los costos de fabricación de los tabiques de arena sílica con los costos de los ofertados en el mercado.

\section{Resultados y análisis de resultados}

En promedio, en la empresa se generan un total de 350 toneladas de arena sílica residual al mes, las cuales son almacenadas para posteriormente ser enviadas a diferentes empresas que las utilizan para diferentes fines. En la Tabla 1 se muestran los resultados obtenidos de las propiedades físicas y mecánicas de la arena sílica.

\begin{tabular}{|l|l|}
\multicolumn{2}{|c|}{$\begin{array}{c}\text { Parámetros de } \\
\text { medición }\end{array}$} \\
\hline Dureza mínima & 7.0 Mohs \\
\hline Estructura & $\begin{array}{l}\text { Monocristalina de alta resistencia a } \\
\text { la abrasión }\end{array}$ \\
\hline Forma & Granos redondos \\
\hline $\begin{array}{l}\text { Contenido de } \\
\text { humedad }\end{array}$ & $<0.1 \% \mathrm{~g} / \mathrm{cm}^{3}$ \\
\hline Peso especifico & $2650 \mathrm{~kg} / \mathrm{m}^{3}$ \\
\hline Densidad aparente & $1476.79 \mathrm{~kg} / \mathrm{m}^{3}$ \\
\hline Densidad a granel & Compactado 98 a $100 \mathrm{~kg} / \mathrm{m}^{3}$ \\
\hline
\end{tabular}

Tabla 1 Características físicas y mecánicas de la arena silica

Fuente: Elaboración Propia.

La dureza mínima de 7.0 Mohs se debe principalmente a la constitución de la arena de sílice, formada en más de un $98 \%$ de cristales de cuarzo, material que se encuentra en abundancia sobre la corteza terrestre y que le da gran estabilidad a la arena haciéndola también insoluble al agua. Debido también a esta composición, se infiere que el residuo presenta altos puntos de fusión y por lo tanto, son ideales para utilizarlos como agregados en las formulaciones para la elaboración de tabicones utilizados en la industria de la construcción, como se pretende en el presente proyecto.

GÓMEZ-BELTRÁN, Guillermina, SIERRA-SALGADO, Diana Vanessa y MARTÍN DEL CAMPO-SÁNCHEZ, Ma. Guadalupe. Elaboración de bloques de construcción a partir de residuos de arena sílica proveniente del proceso de fundición aluminio en una empresa automotriz. Revista de Ingeniería Civil. 2019 
La estructura monocristalina del residuo nos indica que su red es continua y no está interrumpida por bordes en sus partículas, lo que contribuye también a aumentar su dureza. Sus granos presentan una forma redonda, lo que permitió que el material presentara una gran permeabilidad y el proceso de mezclado de las formulaciones se llevara a cabo con facilidad.

El contenido de humedad se determinó por medio de un secado a horno donde la humedad de la muestra de arena es la relación expresada en porcentaje, entre el peso del agua existente y el peso de las partículas sólidas. Se obtuvo un resultado $<0.1 \%$, lo que indica en términos prácticos, que la arena está prácticamente seca, lo cual facilita su manejo. Por otro lado, el peso específico fue de 2650 $\mathrm{kg} / \mathrm{m} 3$ concordante con lo reportado en bibliografía.

En planta Fundición Aluminio existen cinco sistemas de recolección de polvos para la regeneración de arena sílica, tres sistemas correspondientes al área de corazones blocks y dos sistemas para el sistema de corazones cabezas. El laboratorio metalúrgico se encarga de tomar muestras representativas para realizar las pruebas de granulometría y determinar los tamaños del grano (Tabla 2 y 3). Este número se calcula a partir de la distribución de tamaños, que se determina mediante una prueba de tamices ASTM estándar. Los resultados se muestran en números AFS que indican el número de granos por unidad de peso. Los residuos acumulados en los filtros corresponden a los granos finos. Las arenas con tamaños de grano menores a 80 micras y mayores a 100 micras corresponden a las arenas de proceso.

\begin{tabular}{|l|r|}
\hline \multicolumn{1}{|c|}{ Equipo } & No. AFS \\
\hline Polvo de bloques & 98.5 \\
\hline $\begin{array}{l}\text { Recuperación de bloques de arena XB } \\
\text { Poniente }\end{array}$ & 123.35 \\
\hline $\begin{array}{l}\text { Recuperación de bloques de arena XB } \\
\text { Oriente }\end{array}$ & 106.09 \\
\hline $\begin{array}{l}\text { AFS: tamaño de partícula } \\
\text { MESH: tamaño de malla }\end{array}$ &
\end{tabular}

Tabla 2 Resultados Granulométricos Área de corazones blocks

Fuente: Elaboración Propia

\begin{tabular}{|l|r|}
\hline Equipo & No. AFS \\
\hline Polvo de Cabezas & 76.76 \\
\hline Recuperación de arena de Cabezas & 114,62 \\
\hline
\end{tabular}

AFS: tamaño de partícula

MESH: tamaño de malla

Tabla 3 Resultados Granulométricos Área de corazones Cabezas

Fuente: Elaboración Propia

\section{Determinación de contaminantes por aluminio en muestra de arena sílica residual}

Esta prueba se realizó en el laboratorio metalúrgico, para determinar la contaminación por aluminio del residuo de arena sílica, mediante la técnica de espectrofotometría de absorción atómica de llama, utilizando un equipo de Absorción atómica, marca Perkin Elmer, modelo 3100, serie 139649. Los resultados (Tabla a), muestran que el residuo no excede los límites máximos permisibles para los constituyentes tóxicos en el extracto PECT establecidos en la NOM-052-SEMARNAT2005.

\begin{tabular}{|l|l|}
\multicolumn{1}{|c|}{ Parámetro } & \multicolumn{1}{c|}{ Aluminio } \\
\hline Método de prueba & EPA 7000 B \\
\hline Unidad & $\mathrm{mg} / \mathrm{L}$ \\
\hline Resultado & $<2.0000$ \\
\hline L.M. P. & 100.00 \\
\hline L.P.C. & 2.0000 \\
\hline Dictamen & Cumple \\
\hline
\end{tabular}

L.M.P = Límite Máximo Permisible que establece la NOM- 052- SEMARNAT -2005

L.P.C = Límite Práctico de Cuantificación $<=$ Menor al límite

Tabla ¡Error! No hay texto con el estilo especificado en el documento. Resultados de la concentración de aluminio por espectrofotometría de absorción atómica de llama Fuente: Elaboración Propia

Con esta prueba se estableció que el material la arena sílica, a pesar de provenir de un proceso donde se mezcla con aluminio no constituye un riesgo para su utilización al emplearse para la fabricación de tabiques. Sin embargo, es necesario tomar en cuenta que el material proveniente de los colectores presenta riesgos para la salud como se muestra en la figura 1 donde se observan pictogramas referentes al residuo de arena sílica. De esta forma se debe contemplar que al estar en constante contacto con las partículas de arena sílica sigue siendo necesario el uso de un protector respiratorio, para evitar la ingestión del producto por vía oral o respiratoria. 


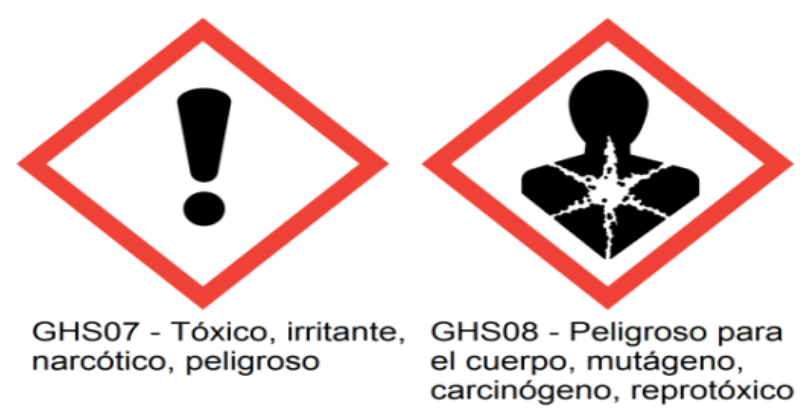

Figura ¡Error! No hay texto con el estilo especificado en el documento. Pictograma referentes al residuo de arena silica

Fuente: NOM-018-STPS-2015

\section{Pruebas CRIT de la arena silica}

Se realizó un análisis CRIT a la arena sílica residual conforme a la normatividad aplicable. Los resultados (Tabla 5) muestran que el residuo no presenta características de peligrosidad. Una ventaja de la propuesta de la elaboración de tabiques es que permite inmovilizar los contaminantes que pudieran estar presentes en las arenas, ya que, al cementar, se impide la lixiviación de los constituyentes potencialmente tóxicos.

\begin{tabular}{|l|l|l|l|}
\hline \multicolumn{5}{|c|}{ Estudios C.R.I.T } \\
\hline $\begin{array}{l}\text { Corrosivid } \\
\text { ad }\end{array}$ & $\begin{array}{l}\text { Reactivid } \\
\text { ad }\end{array}$ & $\begin{array}{l}\text { Tóxico al } \\
\text { ambiente }\end{array}$ & $\begin{array}{l}\text { Inflamabili } \\
\text { dad }\end{array}$ \\
\hline $\begin{array}{l}\text { No es es } \\
\text { corrosivo }\end{array}$ & $\begin{array}{l}\text { No es tóxico } \\
\text { reactivo }\end{array}$ & $\begin{array}{l}\text { No es } \\
\text { inflamable }\end{array}$ \\
\hline
\end{tabular}

Tabla 5 Resultados de la prueba C.R.I.T

Fuente: Elaboración Propia

\section{Elaboración de tabiques}

El proceso de fabricación del molde se realizó de acuerdo con la normatividad NMX-C-404-1997ONNCCE acoplándose a las medidas indicadas, $24 \mathrm{~cm}$ a lo largo, $14 \mathrm{~cm}$ de altitud y $10 \mathrm{~cm}$ de profundidad. Se probaron dos formulaciones para la elaboración de los tabiques siguiendo las especificaciones de la Tabla 6. Cada una de ellas se utilizó para la fabricación de tabiques en pruebas por triplicado.

\begin{tabular}{|l|r|r|}
\cline { 2 - 3 } \multicolumn{1}{c|}{} & Formulación 1 & Formulación 2 \\
\hline Arena Sílica & $20 \mathrm{~kg}$ & $20 \mathrm{~kg}$ \\
\hline Cemento Gris & $2 \mathrm{~kg}$ & $5 \mathrm{~kg}$ \\
\hline Agua & $5 \mathrm{~L}$ & $5 \mathrm{~L}$ \\
\hline Cal & & $2 \mathrm{~kg}$ \\
\hline
\end{tabular}

Tabla 6 Formulaciones para la elaboración de tabicones Fuente: Elaboración Propia
Para iniciar la elaboración de los tabiques se realizó la dispersión de arena sílica sobre el suelo y con la ayuda de una pala recta se dio forma de capuchón para agregar los materiales posteriores a la mezcla. Posteriormente se agregó el cemento gris marca Portland y se mezcló una vez más junto con todos los componentes con ayuda de la pala hasta obtener una mezcla heterogénea. Finalmente, se agregó el agua en proporciones pequeñas hasta que se logró una mezcla compacta entre todos los agregados.

Para la formulación 2, se agregó además la cantidad de cal indicada en la Tabla 6. La mezcla se vertió dentro del molde cubierto en su interior por una ligera capa de aceite quemado de motor, y se comprimió con ayuda de una cuchara hasta lograr acabado rígido y se dejó secar por 4 días protegiéndolo de la lluvia, al cabo de los cuales se desmoldaron las pruebas mecánicas del producto.

\section{Pruebas de compresión y capacidad de absorción}

Los valores de compresión y capacidad de absorción de agua se obtuvieron con base a lo establecido en la NMX-C-404-1997-ONNCCE (Tabla 7). Se observa que los porcentajes son mayores en ausencia de cal, debido a que la capilaridad del tabique es mayor y eso le permite introducir el agua por sus poros. Sumado a ello, la incorporación de cal provoca un fenómeno llamado "Cal apagada" donde en el proceso se desprende gran cantidad de calor que evapora parte del agua utilizada.

\begin{tabular}{|c|c|c|}
\hline Tabique & $\begin{array}{c}\text { Formulación } \\
1\end{array}$ & $\begin{array}{c}\text { Formulación } \\
2\end{array}$ \\
\hline 1 & 11.11 & 8.82 \\
\hline 2 & 10.93 & 8.69 \\
\hline 3 & 10.40 & 8.94 \\
\hline Promedio & $10.813 \pm 0.30$ & $8.816 \pm 0.10$ \\
\hline $\begin{array}{l}\text { Coeficiente } \\
\text { variación }\end{array}$ & $30.14 \%$ & $10.21 \%$ \\
\hline
\end{tabular}

Tabla 7 Capacidad de adsorción (\%)

Fuente: Elaboración Propia

De acuerdo con el análisis propuesto por la NMX-C-036-ONNCCE se realizaron los cálculos de resistencia a la compresión de los tabiques considerando valores mínimos permitidos de $\boldsymbol{f} \boldsymbol{p}^{\prime}$ y $\boldsymbol{f} \boldsymbol{p}$, tomando en cuenta que para fp se deben tomar valores mayores a 90 y para $\mathrm{Cp}$ se debe tomar un valor de 0.35 para formar piezas de producción artesanal.

GÓMEZ-BELTRÁN, Guillermina, SIERRA-SALGADO, Diana Vanessa y MARTÍN DEL CAMPO-SÁNCHEZ, Ma. Guadalupe. Elaboración de bloques de construcción a partir de residuos de arena sílica proveniente del proceso de fundición aluminio en una empresa automotriz. Revista de Ingeniería Civil. 2019 
Los resultados obtenidos (Tabla 8) muestran que la resistencia de diseño (f') se encuentra dentro de los rangos de aceptación según la normatividad aplicada. Se observa que la formulación número dos presenta valores más elevados de resistencia, esto puede representar una característica favorable ya que la presencia de cal en la mezcla que crea una mejor fijación con las partículas de arena, y actúa como absorbente de humedad.

\begin{tabular}{|l|r|r|r|r|r|r|}
\hline & \multicolumn{4}{|c|}{ Formulación 1 } & \multicolumn{4}{|c|}{ Formulación 2 } \\
\hline Tabiqu & $\overline{f_{p}}$ en $\frac{\mathrm{kg}}{\mathrm{cm}}$ & $C_{p}$ & $f_{p e n \frac{\mathrm{kg}}{\mathrm{cm}}}^{\prime}$ & $\overline{f_{p}}$ en $\frac{\mathrm{kg}}{\mathrm{cm}^{2}}$ & $C_{p}$ & $f_{p \text { en } \frac{\mathrm{kg}}{\mathrm{cm}}}^{\prime 2}$ \\
\hline 1 & 200 & .3 & 106.66 & 250 & .3 & 133.33 \\
& & 5 & & & 5 & \\
\hline 2 & 205 & .3 & 109.33 & 255 & .3 & 136 \\
& & 5 & & & 5 & \\
\hline 3 & 210 & .3 & 112 & 258 & .3 & 137.6 \\
& & 5 & & & 5 & \\
\hline
\end{tabular}

Tabla 8 Resistencia a la compression Fuente: Elaboración Propia

\section{Comparación del ladrillo convencional con el de arena silica}

Los ladrillos comerciales son un producto que se ha consumido a lo largo del tiempo para la elaboración de edificaciones, sin embargo, con el paso del tiempo la variedad de los materiales de construcción ha aumentado y con ello la oportunidad de elegir la mejor opción dentro del mercado. A continuación, se muestran algunas características que son consideradas en la elaboración de los tabiques y podría funcionar en la comparación del tabicón artesanal hecho con arena sílica y el comercial (Tabla 9).

\begin{tabular}{|c|c|c|}
\hline Características & Tabicón comercial & $\begin{array}{c}\text { Tabicón de arena } \\
\text { sílica }\end{array}$ \\
\hline Color & Gris opaco & Café grisáceo \\
\hline Peso & $5.93 \mathrm{~kg}$ & $6.8 \mathrm{~kg}$ \\
\hline $\mathrm{pH}$ & 8 & 8 \\
\hline Densidad & $1600 \mathrm{~kg} / \mathrm{m} 3$ & $1300 \mathrm{~kg} / \mathrm{m} 3$ \\
\hline $\begin{array}{ll}\begin{array}{l}\text { Capacidad } \\
\text { absorción }\end{array} & \text { de } \\
\end{array}$ & $10.8 \%$ & $8.821 \%$ \\
\hline $\begin{array}{lll}\begin{array}{l}\text { Resistencia } \\
\text { compresión }\end{array} & \text { a la } \\
\end{array}$ & $100 \mathrm{~kg} / \mathrm{cm} 2$ & $122.48 \mathrm{~kg} / \mathrm{cm} 2$ \\
\hline $\begin{array}{ll}\text { Material } & \text { de } \\
\text { fabricación }\end{array}$ & $\begin{array}{l}\text { Cemento gris, } \\
\text { arena, grava y agua }\end{array}$ & $\begin{array}{l}\text { Cemento gris, arena } \\
\text { sílica, agua y cal }\end{array}$ \\
\hline $\begin{array}{ll}\text { Tiempo } \\
\text { fabricación }\end{array}$ & $7 \mathrm{~min}$ & $12 \mathrm{~min}$ \\
\hline $\begin{array}{l}\text { Tiempo de secado } \\
\text { natural }\end{array}$ & 120 horas & 168 horas \\
\hline Textura & Porosa & Lisa y rasposa \\
\hline
\end{tabular}

Tabla 9 Comparación de algunas características de los tabicones comerciales y el de arena silica

Fuente: Elaboración Propia
Se examinaron varias características que son representativas al momento de comparar un tabicón de construcción, el color del tabique variara de acuerdo con el material del que es fabricado, el peso cambiara de acuerdo con las características porosas del producto, en este caso el peso del tabique fabricado de arena sílica es mayor debido a que las arenas son comprimidas fácilmente y cubren mejor la superficie del molde al ser vertido en su interior, en caso contrario, el peso del tabicón comercial es menor por su nivel de porosidad en las arenas gruesas y ligeras.

El valor de $\mathrm{pH}$ no cambia a causa de que ambos están fabricados con cemento gris, el cual predomina dentro de la mezcla y convierte el material de construcción en un medio básico.

La densidad, como se sabe, es una magnitud escalar que permite medir la cantidad de masa que hay en determinado volumen de una sustancia, la densidad promedio que se obtuvo de ambos tabiques varía de acuerdo con el volumen que se desee cubrir con la mezcla, en este caso la bibliografía nos dice que la densidad de un tabique fabricado con cemento gris, arena, grava y agua es menor al que se obtuvo en las pruebas y cálculos del tabique artesanal de este proyecto.

Los tabiques experimentan un ligero aumento de peso cuando se exponen al ambiente, debido a que absorben humedad del aire. Este efecto es distinto de unos a otros, la porosidad que contenga el material determina el porcentaje de absorción, el tabique fabricado de arena sílica representa un valor menor a la densidad de un tabique comercial, dado que la estructura capilar impide una completa saturación de agua en el tabique.

La resistencia a la compresión de los tabiques depende de los materiales empleados en su fabricación, así como del proceso mismo. Un tabique hecho con pasta aguada puede resistir poco a la compresión, mientras que un tabique de alta resistencia pudiera aguantar hasta 1,400 $\mathrm{kg} / \mathrm{cm} 2$ como marca la bibliografía. Bajo pruebas teóricas el tabique de arena sílica presenta mayor resistencia, comparado con el tabique comercial, esto dependerá según la formulación empleada. 
Actualmente en cualquier fábrica de ladrillos se llevan a cabo una serie de proceso estándar que permite la elaboración en serie de grandes cantidades producto, en este caso los tabiques comerciales pueden elaborarse en menos de 7 minutos con ayuda de una mezcladora, en comparación con los artesanales que tardan aproximadamente 12 minutos desde la elaboración de la mezcla hasta el vertido en el molde.

Una vez que se realiza el vertido de mezcla dentro del molde el tiempo estimado de secado para el tabique comercial es de 120 horas equivalentes a 5 días, y para el tabique artesanal es de aproximadamente 168 horas equivalentes a 7 días, las condiciones meteorológicas influyen en el proceso de secado de cualquier material.

La textura depende de la forma en que están entrelazadas las partículas de un tabique varía según material del que sea fabricado y el moldeo que se realice. La textura del tabique comercial es más granular que la de un ladrillo artesanal, puede que deba al método de moldeo o a la compresión y tiempo de secado del tabique dentro del molde o simplemente el tamaño de sus partículas.

\section{Comparación de costos entre los tabiques comerciales con los de arena sílica residual}

Para la valoración de costos se comparó el costo del tabique de arena sílica con el de 12 tabiques comerciales más consumidos en obras de construcción que incluyeron los fabricados con adobe, arcilla, tierra, refractarios, ligeros y pesados. Los productos para construcción dentro del mercado son muy variados, sin embargo, al realizar una investigación bibliográfica y de campo se observó que los tipos de tabique, blocks y ladrillos cambian su valor de acuerdo con las características del material con los que se elaboran, unos serán más resistentes o ligeros, otros más pesados, lisos o rasposos; estas y otras características definirán la calidad y el costo que se ofrecerá al consumidor.

De esta manera, el block hecho de cerámica, que se fabrica con arcilla y agua, es más caro a diferencia de los demás, y esto se debe a su dificultad de fabricación. También es importante contemplar los usos a los que puede ser destinado. Se pueden utilizar en los adoquines cerámicos, como material de construcción y además en lugares donde se quieren mejorar la pavimentación.
Sin embargo, el precio más alto se da porque el producto tiene una mayor cantidad de materias primas que al mismo tiempo aumenta su valor monetario, pero no quiere decir que el más costoso sea el mejor, siempre se tendrá la opción de comparar, y esto da la oportunidad de equipararlo con el tabique de arena sílica residual, que presenta una resistencia a la compresión de $135.644 \mathrm{~kg} / \mathrm{cm} 2$ que es mayor al de un block cerámico de $70 \mathrm{~kg} / \mathrm{cm} 2$.

Para el cálculo de los costos del tabique de arena sílica se tomó en cuenta el costo del material, su manejo, la mano de obra y una utilidad del $30 \%$, dando un precio de $\$ 9.6 .00$. (Nueve pesos con 06/100 M.N.) Posicionando a este producto en un precio accesible al consumidor. Aun cuando este precio es en promedio $21 \%$ más alto que algunos ladrillos comerciales presenta las ventajas de tener un mayor grado de resistencia a la compresión, menor porcentaje de absorción y sobre todo el aprovechamiento de un desperdicio industrial.

\section{Conclusiones y recomendaciones}

Los resultados obtenidos en el trabajo indican que la utilización de arena residual de fundición como materia prima es factible para la fabricación tabiques de construcción, por sus propiedades físicas y mecánicas, y porque de acuerdo con la prueba CRETI realizada no representa características de peligrosidad. De las formulaciones probadas, los tabiques que incluyen la incorporación de cal muestran mejores propiedades mecánicas. En cuanto a costos, un tabique artesanal de arena sílica entra en el rango de materiales más económicos. Independientemente a dicho costo, se debe priorizar el beneficio ambiental del uso de la arena sílica residual para la fabricación de tabiques $\mathrm{u}$ otros productos como cemento o pavimento.

\section{Referencias}

Herrera, A. E. (2011). Desarrollo de un aglutinante orgánico para la fabricación de moldes y corazones de arena (Tesis Doctoral) Recuperado http://eprints.uanl.mx/2112/6/1080191564.pdf 
Secretaria del Medio Ambiente y Recursos Naturales (2006). Ley General para la Prevención y Gestión Integral de los Residuos. Diario Oficial de la Federación. 8 de octubre del $2003 . \quad$ Recuperado de: http://www.diputados.gob.mx/LeyesBiblio/ref/l gpgir/LGPGIR_orig_08oct03.pdf

Secretaria del Medio Ambiente y Recursos Naturales (2006). Norma Oficial Mexicana NOM-052-SEMARNAT-2005, Que establece las características, el procedimiento de identificación, clasificación y los listados de los residuos peligrosos. Diario Oficial de la Federación. Segunda Sección. 1-32 pp. Recuperado de https://www.sinec.gob.mx/SINEC/Vista/Norma lizacion/DetalleNorma.xhtml?pidn=d3VtZitac2 M4aW1mY1ozRDZmaEM3UT09

Secretaría del Trabajo y Previsión Social. (2015). NORMA Oficial Mexicana NOM-018STPS-2015, Sistema armonizado para la identificación y comunicación de peligros y riesgos por sustancias químicas peligrosas en los centros de trabajo. Diario Oficial de la Federación. 9 de octubre del 2015. Recuperado de:

http://www.dof.gob.mx/nota_detalle.php?codig $\mathrm{o}=5411121 \&$ fech $=09 / 10 / 2015$ 